Zbl Arbeitsmed 2015 - 65:188-189

DOI 10.1007/s40664-015-0040-1

Online publiziert: 16. Juli 2015

๑) Springer-Verlag Berlin Heidelberg 2015

CrossMark

\author{
D.A. Groneberg ${ }^{1}$ M. Jäger ${ }^{2}$ \\ ${ }^{1}$ Institut für Arbeitsmedizin, Sozialmedizin und Umweltmedizin, \\ Goethe-Universität Frankfurt am Main, Frankfurt am Main, Deutschland \\ ${ }^{2}$ IfADo - Leibniz-Institut für Arbeitsforschung, TU Dortmund, Dortmund, Deutschland
}

\title{
Schriftleitung in neuer Formation
}

lag danken den beiden auf das Herzlichste für ihren langjährigen intensiven Einsatz für „unser“ Zentralblatt.

Neben dem bisherigen federführenden Schriftleiter für das Gebiet Medizin, Prof. Dr. David Groneberg (• Abb. 3), wird PD Dr. Matthias Jäger (• Abb. 4) zukünftig für das Gebiet Ergonomie und Arbeitsschutz federführend zuständig sein. Matthias Jäger ist dem Zentralblatt seit langem verbunden, arbeitet im Leibniz-Institut für Arbeitsforschung an der TU Dortmund und vertritt dort in der Lehre das Fach Ergonomie in der Fakultät Maschinenbau. Sein wissenschaftliches Interesse betrifft arbeitsbedingte Belastungen und Erkrankungen des muskuloskeletalen Systems mit besonderem Fokus auf den Rücken und korrespondierenden Möglichkeiten zur Prävention. Prof. Dr. rer. nat. Dipl.-Ing. Alwin Luttmann (• Abb. 2). Schriftleitung und Ver-

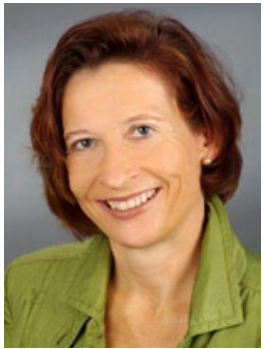

Abb. $1 \Delta$ Prof. Dr. Birgitta Kütting

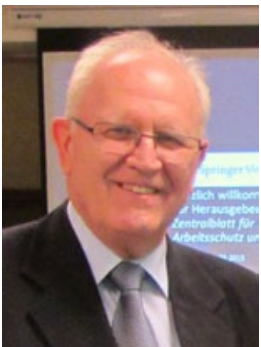

Abb. 2 \ Prof. Dr. Alwin Luttmann

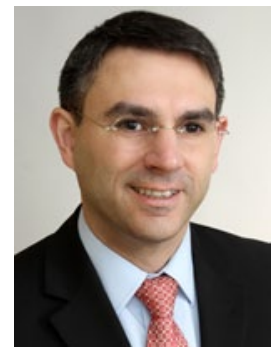

Abb. $3 \Delta$ Prof. Dr. David Groneberg

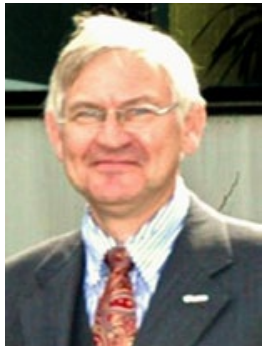

Abb. 4 ^ PD Dr. Matthias Jäger
Für das Gebiet Medizin wird einerseits Prof. Dr. med. Andreas Seidler (• Abb. 5) weiterhin zuständig sein und andererseits zusätzlich Prof. Dr. med. Albert Nienhaus (- Abb. 6). Beide sind dem Zentralblatt als Schriftleiter bzw. Herausgeber seit Jahren verbunden. Prof. Seidler leitet an der TU Dresden das Institut und die Poliklinik für Arbeitsmedizin und Sozialmedizin. Sein Tätigkeitsschwerpunkt liegt im Bereich arbeitsbedingter Erkrankungen und Berufskrankheiten sowie entsprechender epidemiologischer Analysen. Prof. Nienhaus ist in der Berufsgenossenschaft für Gesundheitsdienst und Wohlfahrtspflege für den Fachbereich Gesundheitsschutz zuständig und leitet zudem das Competenzzentrum Epidemiologie und Versorgungsforschung bei Pflegeberufen am Universitätsklinikum Hamburg-Eppendorf.

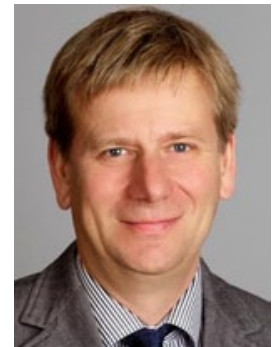

Abb. $5 \Delta$ Prof. Dr. Andreas Seidler

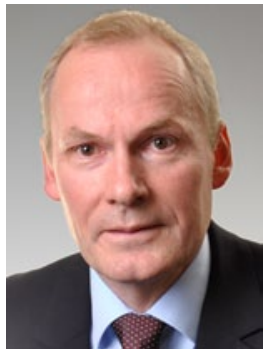

Abb. $6 \Delta$ Prof. Dr. Albert Nienhaus 

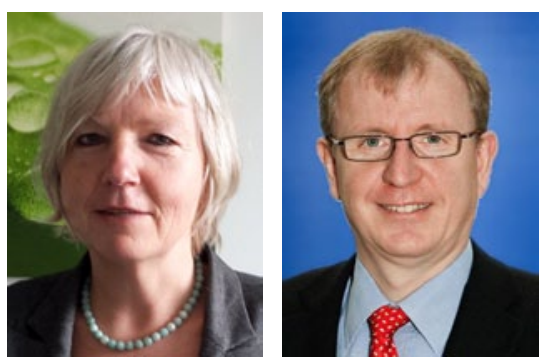

Abb. $7 \Delta$ Prof. Dr. Ute Latza

Abb. $8 \Delta$ Dr.

Klaus Schäfer

Das Gebiet Ergonomie und Arbeitsschutz geht aus dem bisherigen Bereich Technik hervor. Diese Umbenennung lehnt sich stärker an den Titel der Zeitschrift an und verdeutlicht den Fokus zeitgemäßer. Dieses Gebiet wird zukünftig auch von Prof. Dr. rer. nat. Ute Latza (- Abb. 7) sowie Dr. rer.nat. Klaus Schäfer (- Abb. 8) als Schriftleiter betreut - beide sind dem Zentralblatt als Herausgeber bzw. Beirat langjährig verbunden. Prof. Latza ist in der Bundesanstalt für Arbeitsmedizin und Arbeitsschutz in Berlin tätig. Ihr Tätigkeitsfeld umfasst die Prävention arbeitsbedingter Muskel-Skelett- sowie Herz-Kreislauf-Erkrankungen mit besonderem Fokus auf die Kombination der beruflichen und außerberuflichen Faktoren mit multifaktorieller Genese. Dr. Schäfer ist Stellvertretender Präventionsleiter der Berufsgenossenschaft Handelund Warenlogistik (BGHW). Zuvor war er bei der BGHW im Bereich „Physikalische Einwirkungen “ tätig, insbesondere auf dem Gebiet muskuloskeletale Belastungen. In diesem Fachbereich ist er auch heute noch an aktuellen Forschungsprojekten und der Weiterentwicklung geeigneter Beurteilungsverfahren beteiligt.

Wir freuen uns auf die künftige $\mathrm{Zu}$ sammenarbeit in der „neu formierten Hauptschriftleitung“ und begrüßen insbesondere die neue Kollegin und die neuen Kollegen mit einem sehr herzlichen Glückauf!

Prof. Dr. David A. Groneberg

Federführender Hauptschriftleiter

Medizin

PD Dr. Matthias Jäger

Federführender Hauptschriftleiter

Ergonomie und Arbeitsschutz

\section{Korrespondenzadressen}

Prof. Dr. D.A. Groneberg

Institut für Arbeitsmedizin

Sozialmedizin und Umweltmedizin

Goethe-Universität Frankfurt am Main

Theodor-Stern-Kai 7, 60590 Frankfurt am Main

arbsozmed@uni-frankfurt.de

\section{PD Dr. Matthias Jäger}

IfADo - Leibniz-Institut für Arbeitsforschung an der TU Dortmund

Ardeystr. 67

44139 Dortmund

jaeger@ifado.de

\section{Wissenschaftsrat stärkt wissenschaftliche Integrität}

Wissenschaftliche Integrität ist die Grundlage für eine hohe Qualität und die Leistungsfähigkeit des Standorts Deutschland. Der Wissenschaftsrat hat diesbezüglich in einem Positionspapier Empfehlungen zur wissenschaftlichen Integrität verabschiedet, womit der Fokus zu einer umfassenden Kultur der Redlichkeit und Qualität an wissenschaftlichen Einrichtungen erweitert werden soll. Zu den Rahmenbedingungen der Integrität sollen vor allem die Vermittlung guter wissenschaftlicher Praxis, Beratung und Aufklärungsstrukturen in Konfliktfällen sowie eine stärkere Ausrichtung auf Qualität statt Quantität in der gesamten Forschungs- und Publikationspraxis zählen.

Die Empfehlungen des Wissenschaftsrates widmen sich nicht nur gravierenden Fällen des Betrugs, sondern betrachten auch die Grauzone nicht integren Verhaltens. Damit geht der Wissenschaftsrat über den Schwerpunkt von Plagiatsfällen in Doktorarbeiten hinaus. Das Positionspapier beleuchtet Rahmenbedingungen, die wissenschaftliche Integrität erschweren können, wie etwa der Druck für Wissenschaftlerinnen und Wissenschaftler, möglichst viel und in hochrangigen Fachzeitschriften zu veröffentlichen, gepaart mit schwierigen Arbeitsbedingungen sowie starken Abhängigkeiten. Hierbei entstehe insbesondere für den Nachwuchs eine Situation, die integres Handeln gefährden kann.

Laut Wissenschaftsrat sollen Hochschulen und wissenschaftliche Einrichtungen künftig im Umgang mit Verdachtsfällen spezielle Beratung in einer neu zu etablierenden, institutionenübergreifenden Einrichtung erhalten.

Quelle: Wissenschaftsrat, www.wissenschaftsrat.de 